\title{
BMJ Open Consumption of ultra-processed foods and associated sociodemographic factors in the USA between 2007 and 2012: evidence from a nationally representative cross-sectional study
}

To cite: Baraldi LG, Martinez Steele E, Canella DS, et al. Consumption of ultraprocessed foods and associated sociodemographic factors in the USA between 2007 and 2012: evidence from a nationally representative crosssectional study. BMJ Open 2018;8:e020574. doi:10.1136/ bmjopen-2017-020574

- Prepublication history for this paper is available online. To view these files, please visit the journal online (http://dx.doi. org/10.1136/bmjopen-2017020574).

Received 14 November 2017 Revised 5 January 2018 Accepted 31 January 2018

\section{Check for updates}

${ }^{1}$ Department of Nutrition, School of Public Health, University of São Paulo, São Paulo, Brazil

${ }^{2}$ Center for Epidemiological

Studies in Health and Nutrition, University of São Paulo, São

Paulo, Brazil

${ }^{3}$ Department of Applied

Nutrition, Institute of Nutrition,

Rio de Janeiro State University, Rio de Janeiro, Brazil

Correspondence to Dr Carlos Augusto Monteiro; carlosam@usp.br

\section{ABSTRACT}

Objectives To compare ultra-processed food consumption across sociodemographic groups and over time (20072008, 2009-2010, 2011-2012) in the USA.

Design Cross-sectional study.

Setting National Health and Nutrition Examination Survey (NHANES) 2007-2012.

Participants All individuals aged $\geq 2$ years with at least one 24-hour dietary recall were included ( $n=23847$ ).

Main outcome measures Average dietary contribution of ultra-processed foods (expressed as a percentage of the total caloric value of the diet), obtained after classifying all food items according to extent and purpose of industrial food processing using NOVA classification.

Data analysis Linear regression was used to evaluate the association between sociodemographic characteristics or NHANES cycles and dietary contribution of ultra-processed foods.

Results Almost $60 \%$ of calories consumed in the period 2007-2012 came from ultra-processed foods. Consumption of ultra-processed foods decreased with age and income level, was higher for non-Hispanic whites or non-Hispanic blacks than for other race/ethnicity groups and lower for people with college than for lower levels of education, all differences being statistically significant. Overall contribution of ultra-processed foods increased significantly between NHANES cycles (nearly $1 \%$ point per cycle), the same being observed among males, adolescents and high school education-level individuals. Conclusions Ultra-processed food consumption in the USA in the period 2007-2012 was overall high, greater among non-Hispanic whites or non-Hispanic blacks, less educated, younger, lower-income strata and increased across time.

\section{INTRODUCTION}

Ultra-processed food and drink products are packaged formulations resulting from several sequences of industrial processes (hence 'ultra-processed'). They are manufactured mostly or entirely from substances derived from foods and several additives

\section{Strengths and limitations of this study}

- Use of a large, nationally representative sample of the US population, increasing generalisability.

- Unlike most articles which have focused on specific food items such as soft drinks or fast food, our study evaluates the impact of a comprehensive group of products whose consumption is increasing rapidly in most countries.

- Dietary data recall bias may lead to underestimation of ultra-processed food consumption, especially if some individuals tend to under-report these types of food items. Should this under-reporting have increased with time in response to a growing awareness of health effects of ultra-processed foods, this could result in a greater underestimation of ultra-processed foods in later years.

- Information indicative of food processing is not consistently determined for all food items in National Health and Nutrition Examination Survey, which could lead to modest overestimation or underestimation of the consumption of ultra-processed foods.

- Social desirability bias may lead to underestimation of ultra-processed food consumption. Should this under-reporting have increased with time in response to a growing awareness of the health effects of ultra-processed foods, this could result in a greater underestimation of ultra-processed food consumption in later years. Differential social desirability bias across socioeconomic groups (should this exist) could lead to both underestimation or overestimation of the studied associations.

used to imitate sensory properties of foods or to disguise unpalatable aspects of the final product. They typically contain little or no intact foods, and are ready to drink, eat or heat up. 12

Worldwide, the relationship between consumption of specific ultra-processed foods such as soft drinks and diet-related chronic non-communicable diseases is 
well documented. ${ }^{3}{ }^{4}$ Cross-sectional studies have also shown an association between ultra-processed food intake and outcomes such as obesity and metabolic syndrome. ${ }^{5-7}$ Increased risks of obesity, hypertension and dyslipidaemia among higher consumers of ultra-processed foods have also been reported by cohort studies. ${ }^{89}$ Thereupon, the rise in obesity and chronic diseases observed in the USA in the last decades ${ }^{10}{ }^{11}$ may have been triggered by the increase in ultra-processed food and drink product availability $^{1213}$ and its negative impact on dietary quality. Indeed, studies have shown that in the USA, ultra-processed foods are the major dietary contributors of added sugars with the mean added sugars content almost tripling between the first and last quintiles of ultra-processed food consumption ${ }^{114}$ An increase in dietary contribution of ultra-processed foods has also been associated with both a decrease in protein, fibre, vitamins $\mathrm{A}, \mathrm{C}, \mathrm{D}$ and $\mathrm{E}$, zinc, potassium, phosphorus, magnesium and calcium densities, and with an increase of carbohydrate, added sugar and saturated fat contents. ${ }^{14}$

Although the USA has been recognised to be one of the first countries to suffer a meaningful rise in ultra-processed food and drink product market availability, ${ }^{12} 13$ there is a lack of evidence regarding recent consumption changes and also differences among sociodemographic strata. Thus, the aim of this study was to compare ultra-processed food consumption across different sociodemographic groups of the US population and to describe the recent evolution of ultra-processed food consumption in this population.

\section{METHODS}

\section{Study population and data collection}

This study included data from three cycles of National Health and Nutrition Examination Survey (NHANES): 2007-2008, 2009-2010 and 2011-2012. NHANES is a continuous, nationally representative, cross-sectional survey of non-institutionalised, civilian US residents. ${ }^{15}$ NHANES sample was obtained using a complex, multistage, probability sampling design.

In the first step of the survey, all participants receive an interviewer at home and complete questionnaires about health history, housing characteristics and family demographic background. Within 1 or 2 weeks, physical examinations and laboratory studies are performed at a mobile examination centre (MEC). During these examinations, participants complete a dietary interview component that includes one 24-hour dietary recall. A follow-up dietary recall is carried out 3-10 days after the MEC examination with all examinees by telephone. Both dietary recalls are administered by trained interviewers using US Department of Agriculture Automated Multiple-Pass Method. Portion sizes were estimated by NHANES as further explained in NHANES Manual. ${ }^{16}$ Shortly, each MEC dietary interview room contains a standard set of measuring guides that are used to help the respondent report the volume and dimensions of the food items consumed. On completion of the in-person interview, participants are given measuring cups, spoons, a ruler and a food model booklet to use for reporting food amounts during the telephone interview.

For children under 9 years of age, the interview was conducted with a proxy; for children between 6 and 8 years of age, in the presence of the child. Children aged 9-11 years provided their own data assisted by an adult household member (assistant). The preferred proxy/ assistant was the most knowledgeable person about the child's consumption on the day before the interview. If the child had more than one caregiver, several individuals could contribute to the intake data.

Of the 10149 people screened in NHANES 2007-2008, $9255(91.2 \%)$ participated in the dietary interview in the MEC and 7838 (77.2\%) answered the follow-up dietary recall. Similarly, in the second NHANES cycle (20092010) of 10537 people screened, $9754(92.6 \%)$ and 8406 $(79.8 \%)$ completed the first and the follow-up dietary recall, respectively. In 2011-2012, of the 9756 individuals screened, $8519(87.3 \%)$ responded to the first dietary recall and $7605(78.0 \%)$ completed the second dietary recall.

In our study, all individuals aged $\geq 2$ years - who completed at least one dietary recall-were considered eligible. Pregnant women and breastfeeding mothers were excluded, resulting in a final study sample of 23847 individuals. For adjusted analyses, we evaluated those individuals who had complete information on sociodemographic variables. Since 4307 participants had missing values on family income and/or educational attainment, multivariable-adjusted analysis included 19540 individuals. These individuals with missing values had similar sociodemographic characteristics (gender, age, race/ ethnicity) and similar mean consumption of ultra-processed foods to the full sample of interviewed participants aged $\geq 2$ years.

\section{Sociodemographic variables}

The sociodemographic variables considered in this study were: gender (male and female); age group (2-9 years, 10-19 years, 20-39 years, 40-59 years, $60+$ years); race/ ethnicity (American non-Hispanic white, American non-Hispanic black, Mexican-American, other Hispanic and other race, including Asians and multiracial). Educational attainment of respondents for participants aged more than 24 years and of household reference person otherwise (less than high school, high school and college). There were 113 households ( $1.51 \%$ of the sample) where the reference person was younger than 25 years old. In these households, the education achievement of the interviewee (in all cases aged between 18 and 24 years) was adopted.

Ratio of family income to poverty categorised based on Supplemental Nutrition Assistance Program eligibility (low, 0.00-1.30; medium, >1.30-3.50 and high, >3.50 and above). ${ }^{15}$ Income-to-poverty ratio is defined as the ratio of family or unrelated individual income to their 
appropriate poverty threshold. Ratios below 1.00 indicate that the income for the respective family or unrelated individual is below the official definition of poverty, while a ratio of 1.00 or greater indicates income above the poverty level'. ${ }^{17}$

\section{Food classification according to processing}

All recorded food items from the three NHANES cycles were classified according to NOVA (a name, not an acronym), a food classification based on the extent and purpose of industrial food processing. ${ }^{2}$ NOVA includes four groups: 'unprocessed or minimally processed foods' (such as fresh, dry or frozen fruits or vegetables; packaged grains and pulses; grits, flakes or flours made from corn, wheat or cassava; pasta, fresh or dry, made from flours and water; eggs; fresh or frozen meat and fish and fresh or pasteurised milk); "processed culinary ingredients' (including sugar, oils, fats, salt and other substances extracted from foods and used in kitchens to season and cook unprocessed or minimally processed foods and to make culinary preparations), 'processed foods' (including canned foods, sugar-coated dry fruits, salted meat products, cheeses and freshly made unpackaged breads, and other ready-to-consume products manufactured with the addition of salt or sugar or other substances of culinary use to unprocessed or minimally processed foods) and 'ultra-processed foods'.

Ultra-processed foods, of particular interest in this study, include soft drinks, sweet or savoury packaged snacks, confectionery and industrialised desserts, mass-produced packaged breads and buns, poultry and fish nuggets and other reconstituted meat products, instant noodles and soups, and many other ready-to-consume formulations of several ingredients. Besides salt, sugar, oils and fats, these ingredients include food substances not commonly used in culinary preparations, such as modified starches, hydrogenated oils, protein isolates and classes of additives whose purpose is to imitate sensorial qualities of unprocessed or minimally processed foods and their culinary preparations, or to disguise undesirable qualities of the final product. These additives include colourants, flavourings, non-sugar sweeteners, emulsifiers, humectants, sequestrants, and firming, bulking, de-foaming, anticaking and glazing agents. Unprocessed or minimally processed foods represent a small proportion of or are even absent from the list of ingredients of ultra-processed products. The rationale underlying the classification, a detailed definition of each NOVA food group and examples of food items classified in each group has been previously published. ${ }^{1}$

For all food items (Food Codes) judged to be a handmade recipe, the classification was applied to the underlying ingredients (Standard Reference Codes or SR Codes) obtained from the US Department of Agriculture (USDA) Food and Nutrient Database for Dietary Studies (FNDDS 4.0, 5.0 and 6.6). For example, for cakes, cookies or pies underlying SR Codes were used unless underlying SR Codes or ingredients were unlikely to be used in home recipe (ie, 'cellulose (alpha-cellulose, powdered cellulose and poly-cellulose)' or 'oil, industrial, soy (partially hydrogenated), multiuse for non-dairy butter flavour' or 'shortening, industrial, soybean (hydrogenated) and cottonseed', or 'whey, sweet, dried').

Other examples are 'salsa, red, cooked, not homemade' and 'salsa, red, cooked, homemade'. Food Code 'salsa, red, cooked, not homemade' was classified as ultra-processed based on information from a similar product included in the Food Code website ('Red Gold Salsa, Mild': tomato concentrate (water, tomato paste), diced tomatoes, jalapeno peppers, green chiles, yellow chiles, vinegar, salt, dried onion, dried garlic, cilantro, natural flavour). On the other hand, for Food Code 'salsa, red, cooked, homemade' it was each underlying SR Code (salt, table; garlic, raw; onions, raw; tomatoes, red, ripe, canned, packed in tomato juice; peppers, hot chiles, sun dried; water, tap, drinking; vegetable oil) instead, that was classified according to NOVA, as further explained in a previously published paper. $^{1}$

\section{Assessing energy content}

For this study, Food code energy values were used as provided by NHANES.

For handmade recipes, the underlying ingredient (SR Code) energy values were calculated using variables from both FNDDS (4.0, 5.0 or 6.6) and USDA National Nutrient Database for SR, Release 23, 24 or 25 (SR23, SR24 or SR25) depending on the NHANES cycle.

\section{Data analysis}

Dietary intake was assessed using means of both recall days when available and 1 day otherwise.

Food items were sorted into mutually exclusive food subgroups within unprocessed or minimally processed foods ( $\mathrm{n}=11$ subgroups), processed culinary ingredients $(n=4)$, processed foods $(n=5)$ and ultra-processed foods $(n=11)$, as shown in table 1 . The dietary intake was described according to the average absolute (total daily energy intake) and relative (expressed as a percentage of the total caloric value of the diet) consumption, provided by each NOVA food group and subgroups within these groups.

Crude and multivariate-adjusted linear regressions were used to evaluate the association between sociodemographic characteristics and dietary contribution of ultra-processed foods. Tests of linear trend were performed in order to assess the effect of age, education and income as single continuous variables. Linear regression was also used to assess how the mean dietary share of ultra-processed foods varied across the three studied cycles, both overall and according to age group, gender, education, income and ethnicity. In order to estimate the evolution of ultra-processed food consumption, Wald tests were performed to compare 2007-2008 and 2011-2012 values. Interaction tests were used to explore differential effects of time across sociodemographic variables. Tests of linear trend across the whole period were also performed. 
Open Access

Table 1 Distribution of daily energy intake according to NOVA food groups

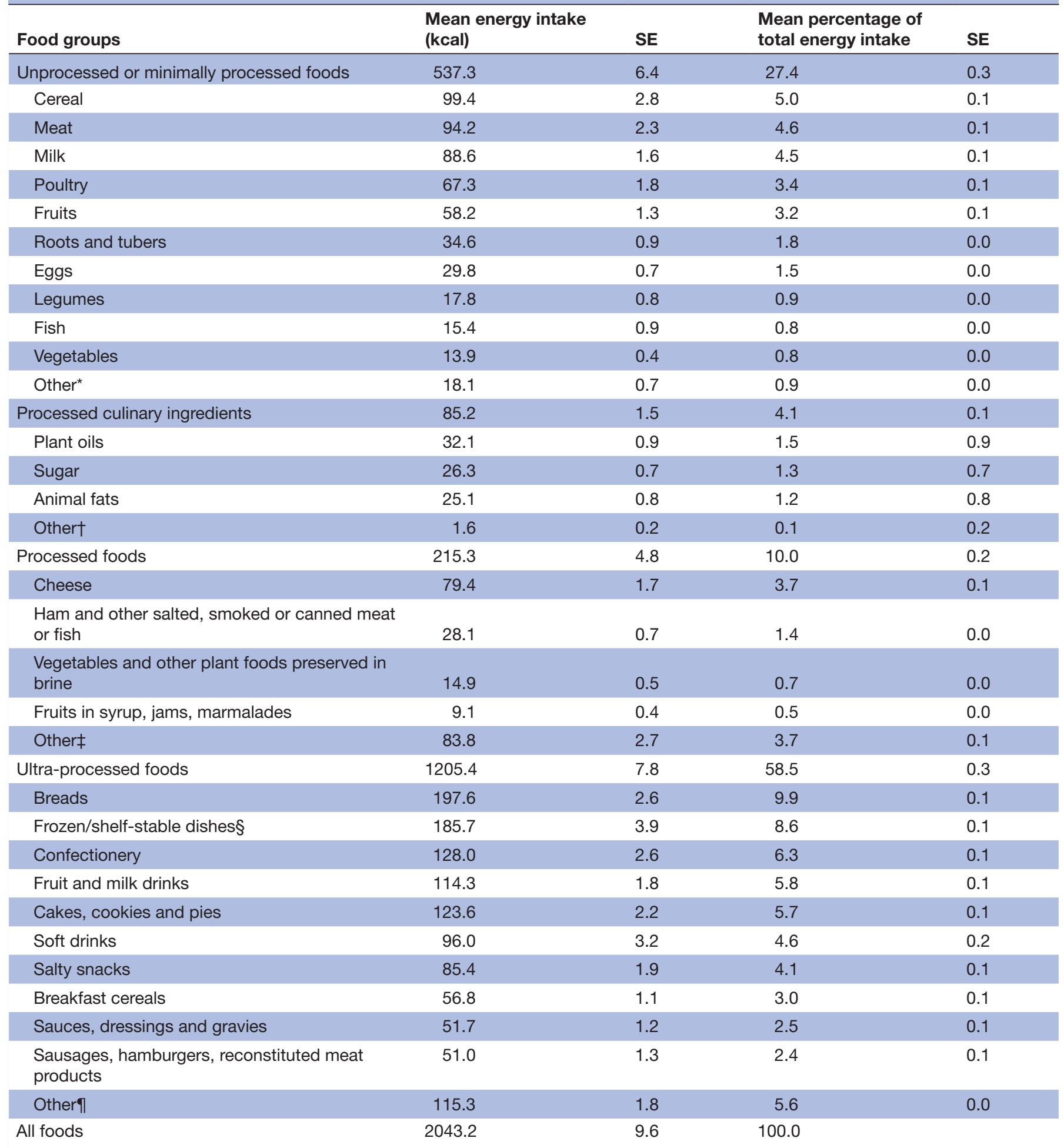

US population aged $\geq 2$ years (NHANES 2007-2012).

*Including nuts and seeds (unsalted); yeast; dried fruits (without added sugars) and vegetables; non-presweetened, non-whitened, non-flavoured coffee and tea; coconut water and meat; homemade soup and sauces; flours; tapioca.

†Including starches; coconut and milk cream; unsweetened baking chocolate, cocoa powder and gelatin powder; vinegar; baking powder and baking soda.

łIncluding salted or sugared nuts and seeds; peanut, sesame, cashew and almond butter or spread; beer and wine.

§Including pizzas and ready-to-eat dishes.

IIncluding soy products such as meatless patties and fish sticks; baby food and baby formula; dips, spreads, mustard and catsup; margarine; sugar substitutes, sweeteners and all syrups (excluding 100\% maple syrup); distilled alcoholic drinks.

NHANES, National Health and Nutrition Examination Survey. 
NHANES sample weights were used in all analyses to account for differential probabilities of selection for the individual domains, non-response to survey instruments and differences between the final sample and the total US population. The Taylor series linearisation variance approximation procedure was used for variance estimation, in order to account for the complex sample design and the sample weights. ${ }^{15}$

Statistical hypotheses were tested using a two-tailed $\mathrm{P}<0.05$ level of significance. Data were analysed using Stata/SE statistical software package version 14.1.

\section{RESULTS}

\section{Distribution of total energy intake according to NOVA food} groups

The average US daily energy intake in 2007-2012 was $2042.5 \mathrm{kcal}$, and most calories (58.5\%) came from ultra-processed foods. Unprocessed or minimally processed foods contributed $27.5 \%$ of total energy, processed foods an additional $10.0 \%$ and processed culinary ingredients the remaining $4.0 \%$ (table 1 ).

Within ultra-processed foods, most calories came from breads and frozen/shelf-stable meals $(9.9 \%$ and $8.6 \%$ of total daily intake, respectively), followed by confectionery $(6.1 \%)$, fruit and milk drinks $(5.8 \%)$, cakes, cookies and pies $(5.7 \%)$, soft drinks $(4.6 \%)$, salty snacks $(4.1 \%)$ and breakfast cereals $(3.0 \%)$ (table 1$)$.

All together food of animal origin accounted for more than a half of calories of unprocessed or minimally processed foods (approximately 15\% of total calories), with milk corresponding to $4.7 \%$ of total calories, meat $4.5 \%$, poultry $3.4 \%$ and eggs $1.5 \%$. Among processed foods, most calories came from cheese (3.7\% of daily calories) and ham or other salted, smoked or canned meat or fish $(1.4 \%)$. Plant oils (1.5\%) and table sugar (2.3\%) were the highest calorie contributors among processed culinary ingredients (table 1).

\section{Association of sociodemographic characteristics with the dietary contribution of ultra-processed foods}

Except for gender, all other sociodemographic characteristics were associated with the dietary contribution of ultra-processed foods. Both the crude and adjusted contribution decreased with age, education and income. The non-adjusted contribution was highest among non-Hispanic blacks (62.1\% of total energy intake), followed by non-Hispanic whites (59.2\%), Mexican-American (57.7\%), other Hispanic (53.5\%) and other (49.9\%). Adjusted estimates indicated similar consumption among non-Hispanic black and non-Hispanic white but did not change substantially the consumption of the other ethnic groups (table 2).

\section{Time changes in the dietary contribution of ultra-processed foods}

As shown in table 3 , the overall dietary contribution of ultra-processed foods linearly increased across the three cycles $(\mathrm{P}<0.05)$ : from $57.6 \%$ in $2007-2008$ to $59.7 \%$ in 2011-2012 (nearly $+1 \%$ per cycle). The same statistically significant linear time trend was found for men, adolescents and respondents with high school education. These linear trends remained unchanged after adjusting for sociodemographic variables (data not shown). Furthermore, when comparing the last cycle against the first one, an increase in the consumption of ultra-processed foods was observed for all sociodemographic strata, ranging from a minimum of $+1.4 \%$ (in high income/poverty family ratio) and a maximum of $+3.6 \%$ (in other race). Significant interaction terms were found for gender-year and age-year $(\mathrm{P}<0.05)$.

\section{DISCUSSION}

This study of recent and representative data of US population shows that ultra-processed foods represented nearly $60 \%$ of dietary calories over a 6 -year period. Small differences in consumption within the population reflect how ultra-processed foods have permeated and reached all social strata, modifying eating behaviours by displacing handmade meals and turning ultra-processed food consumption into an important eating pattern. Individuals with a college education consumed the least ultra-processed foods whereas adolescents and American black and white ethnic groups were the highest consumers. Ultra-processed food consumption was inversely associated with both age and income levels and did not vary according to sex.

There was an overall time trend increase in ultra-processed consumption between 2007-2008 and 2011-2012, and more specifically among men, adolescents and individuals with high school education. Among middle income-to-poverty ratio individuals and non-Hispanic white Americans, an increase in ultra-processed food consumption was observed between the first and last cycles.

Population-based studies evaluating ultra-processed food consumption according to sociodemographic variables carried out in different countries obtained similar results. In the UK (2008-2009), the average ultra-processed food consumption was $53 \%$ of total energy intake and decreased with age (from 58.2\% among 18-29 years to $50.6 \%$ among $70+$ years). ${ }^{18}$ In Canada (2004), the average consumption of ultra-processed foods was $47.7 \%$ remaining high in all socioeconomic groups, especially among children and adolescents $(55.1 \%)$ and less educated individuals $(51.7 \%) .{ }^{19}$ In Chile (2010) and Mexico (2012), ultra-processed food consumption was much lower than in USA-28.6\% and $29.8 \%$, respectively-and also decreased with age. ${ }^{2021}$ In Chile, ultra-processed food consumption increased with family income from $25.8 \%$ to $30.1 \%$ of total energy intake. ${ }^{20}$ In Mexico, ultra-processed food consumption increased with socioeconomic status (SES). ${ }^{21}$ In a cohort study carried out in France (including participants recruited between 2009 and 2014), ultra-processed foods contributed $35.9 \%$ of 
Table 2 Dietary contribution of ultra-processed foods according to sociodemographic variables

$\%$ of energy intake from ultra-

processed foods

\begin{tabular}{|c|c|c|}
\hline Variables & Crude mean $(95 \% \mathrm{Cl})$ & Adjusted mean† $(95 \% \mathrm{Cl})$ \\
\hline \multicolumn{3}{|l|}{ Gender } \\
\hline Male & 58.4 (57.6 to 59.1$)$ & 58.3 (57.6 to 59.0$)$ \\
\hline Female & 58.6 (57.9 to 59.4$)$ & 58.8 (58.1 to 59.5$)$ \\
\hline \multicolumn{3}{|l|}{ Age group } \\
\hline $2-9$ & 65.2 (64.3 to 66.1$)$ & 65.9 (65.0 to 66.8$)$ \\
\hline $10-19$ & 66.7 (65.9 to 67.5$)$ & 66.8 (65.9 to 67.7$)$ \\
\hline 20-39 & 59.0 (58.0 to 60.1$)$ & 59.5 (58.7 to 60.3 ) \\
\hline $40-59$ & 55.2 (54.0 to 56.3$)$ & 55.2 (54.1 to 56.4$)$ \\
\hline$\geq 60$ & $53.5(52.5 \text { to } 54.4)^{*}$ & $52.8(51.9 \text { to } 53.7)^{*}$ \\
\hline \multicolumn{3}{|l|}{ Education $\ddagger$} \\
\hline Less than high school & 59.0 (57.9 to 60.0$)$ & 59.5 (58.4 to 60.6$)$ \\
\hline High school & 59.9 (59.2 to 60.6$)$ & 59.7 (59.1 to 60.3) \\
\hline College or higher & $55.4(54.2 \text { to } 56.8)^{*}$ & $55.9(54.6 \text { to } 57.2)^{*}$ \\
\hline \multicolumn{3}{|c|}{ Family income-to-poverty ratio§ } \\
\hline$\leq 1.30$ & 61.1 (60.0 to 62.2) & 59.6 (58.6 to 60.7$)$ \\
\hline $1.31-1.50$ & 58.9 (57.9 to 59.9$)$ & 58.7 (57.8 to 59.7$)$ \\
\hline$>1.50$ & $56.8(55.9 \text { to } 57.8)^{*}$ & $57.7(56.9 \text { to } 58.6)^{*}$ \\
\hline \multicolumn{3}{|l|}{ Ethnicity } \\
\hline Non-Hispanic white & 59.2 (58.4 to 60.1$)$ & 60.2 (59.4 to 60.9$)$ \\
\hline Non-Hispanic black & 62.1 (61.1 to 63.3) & 60.6 (59.7 to 61.5$)$ \\
\hline Mexican-American & 57.7 (56.7 to 58.8$)$ & 54.8 (53.2 to 56.3 ) \\
\hline Other Hispanic & 53.3 (51.5 to 55.1$)$ & $52.0(50.3$ to 53.7$)$ \\
\hline Other & 49.9 (47.7 to 52.2) & $49.6(47.3 \text { to } 51.8)^{*}$ \\
\hline
\end{tabular}

US population aged $\geq 2$ years (NHANES 2007-2012).

${ }^{*} \mathrm{P}$ for linear trend $<0.05$.

†Adjusted for all the other variables in the table.

fFor individuals under 25 years, the education of the household head was considered.

$\S$ Income-to-poverty ratios represent the ratio of family or unrelated individual income to their appropriate poverty threshold. Categories based on SNAP eligibility. ${ }^{38}$

NHANES, National Health and Nutrition Examination Survey; SNAP, Supplemental Nutrition Assistance Program.

total energy intake and consumption was higher among younger aged and lower educated individuals. ${ }^{22}$

While our study observed an increase in ultra-processed food consumption between 2007 and 2012, an investigation using the alternative index of diet quality showed an increase in US adult diet quality between 1999 and 2012. ${ }^{23}$ This stands in apparent contradiction if we take into account results from studies on NHANES food intake and on national US household food purchases. Indeed, these studies have demonstrated that the dietary contribution of ultra-processed foods is inversely associated with the dietary content of protein, fibre, and most micronutrients and directly associated with carbohydrate, saturated fat, total sugar, added sugar and sodium contents. ${ }^{14} 24$ Still, it must be noted that more than $50 \%$ of the improvement in AHEI-2010 overall score was attributed to a reduction in trans fat intake, ${ }^{25}$ which is not inconsistent with an increasing consumption of ultra-processed foods, especially in view of the reduction in trans fat use in industrial products. The limits of ultra-processed food reformulation have been discussed elsewhere. ${ }^{26}$

The negative gradient between age group and ultra-processed food consumption is consistent with the significant positive association observed between age and dietary quality in US adults from 1999 to $2010 .^{23}$ This negative gradient may be shaped by differential age-dependent food preferences. ${ }^{27}$ Indeed, children and adolescents may have a higher demand of ultra-processed foods because of a greater promotion through marketing and advertising among these age groups, ${ }^{28}$ and also availability in school food environment. The influence of the school food environment, including school vending machines and school stores/canteens/snack bars, on children and adolescents' dietary intake is well known. ${ }^{29}$ Indeed, in recent years, the healthiness of diets among children 
Table 3 Time changes in the dietary contribution of ultra-processed foods (\% or total energy intake) according to sociodemographic variables

\begin{tabular}{|c|c|c|c|c|}
\hline Variables & 2007-2008 & 2009-2010 & 2011-2012 & $\begin{array}{l}\text { P value for linear } \\
\text { trend }\end{array}$ \\
\hline \multicolumn{5}{|l|}{ Gender } \\
\hline Male & 57.3 & 58.8 & 59.7 & 0.0368 \\
\hline Female & 57.9 & 58.9 & 59.6 & 0.1834 \\
\hline \multicolumn{5}{|l|}{ Age group } \\
\hline $2-9$ & 63.4 & 63.9 & 65.4 & 0.4518 \\
\hline $10-19$ & 64.9 & 67.0 & 68.3 & 0.0128 \\
\hline 20-39 & 58.0 & 59.2 & 59.9 & 0.3529 \\
\hline $40-59$ & 54.2 & 55.0 & 56.3 & 0.3821 \\
\hline$\geq 60$ & 52.1 & 54.2 & 54.0 & 0.1800 \\
\hline \multicolumn{5}{|l|}{ Education } \\
\hline Less than high school & 58.0 & 58.6 & 60.4 & 0.1632 \\
\hline High school & 58.4 & 60.2 & 61.1 & 0.0122 \\
\hline College & 54.4 & 55.9 & 56.0 & 0.4667 \\
\hline \multicolumn{5}{|c|}{ Family income-to-poverty ratio $†$} \\
\hline$\leq 1.30$ & 60.6 & 60.9 & 62.6 & 0.1910 \\
\hline $1.31-3.50$ & 57.7 & 59.5 & 60.1 & 0.0380 \\
\hline$>3.50$ & 56.1 & 57.4 & 57.5 & 0.2310 \\
\hline \multicolumn{5}{|l|}{ Ethnicity } \\
\hline Non-Hispanic white & 57.9 & 59.4 & $60.4^{*}$ & 0.0749 \\
\hline Non-Hispanic black & 61.6 & 61.1 & 63.6 & 0.1512 \\
\hline Mexican-American & 56.4 & 58.4 & 58.3 & 0.0501 \\
\hline Other Hispanic & 51.4 & 55.3 & 53.1 & 0.2563 \\
\hline Other Race & 47.4 & 50.5 & 51.0 & 0.4002 \\
\hline Total & 57.6 & 58.9 & 59.7 & 0.038 \\
\hline
\end{tabular}

US population aged $\geq 2$ years (NHANES 2007-2012).

${ }^{*} \mathrm{P}<0.05$ Wald test for difference in consumption between the first (2007-2008) and the last (2011-2012) cycles.

†Income-to-poverty ratios represent the ratio of family or unrelated individual income to their appropriate poverty threshold. Categories based on SNAP eligibility. ${ }^{38}$

NHANES, National Health and Nutrition Examination Survey; SNAP, Supplemental Nutrition Assistance Program.

and adolescents are the ones to have declined the most. Snacks, pizzas, pastries, sweetened fruit juices and ready-made Mexican dishes (mixed dishes with corn or flour tortillas and corn-based dishes), most of them ultra-processed foods, were the ones to present the greatest caloric intake increase in the last 20 years among American children aged 2-6 years. ${ }^{30}$ In addition, in the last decades among adolescents, a total of $17 \%$ of all calories came from fast food. ${ }^{24}$ All these previous findings are consistent with our findings of higher ultra-processed food consumption among younger age groups and positive time trend in ultra-processed food intake among adolescents.

Although some studies have reported that non-Hispanic black Americans have less favourable dietary patterns than non-Hispanic white Americans,${ }^{31}$ in our study both groups presented the highest consumption of ultra-processed foods. A previous study also showed that in most NHANES survey cycles between 1999 and 2010, non-Hispanic whites had a significantly lower dietary quality than Mexican-Americans adults. ${ }^{23}$ A possible explanation for differences between non-Hispanic white and non-Hispanic black Americans and the remaining ethnicities may be greater promotion and advertising of ultra-processed foods to these race/ethnicities. ${ }^{32}$ Differences may also arise from the fact that households with foreign-born reference persons tend to cook more dinners at home than those with US-born reference person ${ }^{32}$ perhaps in an attempt to maintain their culinary traditions or out of simple routine and not having been fully acculturated to the fast food culture yet. However, it is known that throughout the process of acculturation (at least among Mexicans), migrants progressively incorporate negative eating habits and with the passing of the generations end up adhering to the North American diet. ${ }^{33}$ 
The small inverse association between education and ultra-processed food consumption is consistent with previous findings of direct association between education and dietary quality of adults in all cycles between 1999 and 2010. ${ }^{23}$ Increased knowledge about nutrition and concern with health may lead individuals to value nutrition over taste and convenience when choosing foods among more highly educated individuals. ${ }^{27}$

Also for income, the small inverse association with ultra-processed food consumption is consistent with previous findings of direct association between income and dietary quality (AHEI-2010) of adults from 1999 to $2010 .{ }^{23}$ On the other hand, the fact that only a small negative gradient was found between SES and ultra-processed food consumption, counters the stereotype that lower-income individuals are higher consumers of ultra-processed foods because of price. As previously highlighted in others studies eating 'junk food' is not necessarily cheaper than eating 'real food' ${ }^{34-36}$ Our results are also consistent with those from a recent US study showing little evidence of a gradient in adult fast-food consumption in regards to income/wealth. ${ }^{27}$ If consumer's food choices should depend on price, income, household manager's time and preferences, ${ }^{37}$ our study shows that the cumulative effect of all four did not lead to an important differential consumption of ultra-processed foods across income strata.

SES disparities in health are thought to be partially caused by an SES gradient in nutrition ${ }^{23} 31$ Our study suggests that a gradient in ultra-processed food consumption is probably not the main or only underlying reason for these health inequities. Indeed, ultra-processed food consumption was high overall, despite being slightly higher among less educated, younger, lower income and American non-Hispanic black and non-Hispanic white strata. Healthier eating should be encouraged among all sociodemographic strata especially by promoting healthier food environments (in schools among others) and regulating marketing. ${ }^{28} 29$

The probabilistic nature of the studied sample and the national representativeness of the American population are strengths of this study. Study limitations should be considered. Recall bias may lead to underestimation of ultra-processed food consumption, especially if some individuals tend to under-report these types of food items. Should this under-reporting have increased with time in response to a growing awareness of health effects of ultra-processed foods, this could result in a greater underestimation of ultra-processed foods in later years. Still, recall bias should be minimised through the use of the five-step validated USDA's Automated Multiple-Pass Method. As NHANES was not specifically designed to classify food items according to degree of processing, misclassification errors may lead to underestimation or overestimation of ultra-processed food consumption. Cases of classification uncertainty were solved using a conservative approach, opting for the lesser degree of processing or assuming a homemade recipe, which could have led to underestimation of ultra-processed food consumption.

Social desirability bias may lead to underestimation of ultra-processed food consumption. Should this under-reporting have increased with time in response to a growing awareness of the health effects of ultra-processed foods, this could result in a greater underestimation of ultra-processed food consumption in later years. Differential social desirability bias across socioeconomic groups (should this exist) could lead to both underestimation or overestimation of the studied associations.

Even though data from two 24-hour dietary recalls may not represent the usual diet of individuals, these data can be useful to estimate group means as was done in this study. Though analyses were controlled for several sociodemographic variables, residual confounding by variables such as region or urban/rural area is always possible.

\section{CONCLUSION}

In this study, we show that ultra-processed food consumption in the USA in the period 2007-2012 was overall high, greater among less educated, younger, lower income and American non-Hispanic black and non-Hispanic white strata, and increased across time. Healthier eating should be promoted among all sociodemographic groups, especially among children and adolescents, which have been shown to be the highest consumers of ultra-processed foods in several countries.

Contributors CAM and LGB designed the research. LGB and EMS took care of data management. LGB, EMS and DSC analysed the data. LGB, EMS and CAM wrote the paper. CAM and LGB had primary responsibility for the final content. All authors revised critically the final version to be published, had full access to all of the data (including statistical reports and tables), read and officially approved the final version. All authors absolutely agreed to be accountable for all aspects of the study.

Funding This work was supported by Conselho Nacional de Desenvolvimento Científico e Tecnológico, Edital MCTI/CNPq/Universal (Processo CNPq number 443477/2014-0) and from Fundação de Amparo à Pesquisa do Estado de São Paulo (Processo FAPESP number 2015/14900-9).

Competing interests None declared.

Patient consent Obtained.

Ethics approval Secondary publicly available data from NHAES were used in this study. NHANES obtained Ethics Committee/Institutional Review Board approval by NCHS Research Ethics Review Board under Continuation of Protocol \#2005-06 and Protocol \#2011-17 for 2007-2008/2009-2010 cycles and for 2011-2012 cycle, respectively.

Provenance and peer review Not commissioned; externally peer reviewed.

Data sharing statement This study is based on open data of the American population that is available by Centers for Disease Control and Prevention in their website: https://www.cdc.gov/nchs/nhanes/index.htm.

Open Access This is an Open Access article distributed in accordance with the Creative Commons Attribution Non Commercial (CC BY-NC 4.0) license, which permits others to distribute, remix, adapt, build upon this work non-commercially, and license their derivative works on different terms, provided the original work is properly cited and the use is non-commercial. See: http://creativecommons.org/ licenses/by-nc/4.0/

(c) Article author(s) (or their employer(s) unless otherwise stated in the text of the article) 2018. All rights reserved. No commercial use is permitted unless otherwise expressly granted. 


\section{REFERENCES}

1. Martínez Steele E, Baraldi LG, Louzada ML, et al. Ultra-processed foods and added sugars in the US diet: evidence from a nationally representative cross-sectional study. BMJ Open 2016;6:e009892.

2. Monteiro CA, Cannon G, Moubarac JC, et al. The UN Decade of Nutrition, the NOVA food classification and the trouble with ultraprocessing. Public Health Nutr 2018.21.

3. Micha R, Khatibzadeh S, Shi P, et al. Global, regional and national consumption of major food groups in 1990 and 2010: a systematic analysis including 266 country-specific nutrition surveys worldwide. BMJ Open 2015;5:e008705.

4. Singh GM, Micha R, Khatibzadeh S, et al. Global, regional, and national consumption of sugar-sweetened beverages, fruit juices, and milk: a systematic assessment of beverage intake in 187 Countries. PLoS One 2015;10:e0124845.

5. Louzada ML, Baraldi LG, Steele EM, et al. Consumption of ultraprocessed foods and obesity in Brazilian adolescents and adults. Prev Med 2015;81:9-15.

6. Canella DS, Levy RB, Martins AP, et al. Ultra-processed food products and obesity in Brazilian households (2008-2009). PLoS One 2014;9:e92752.

7. Tavares LF, Fonseca SC, Garcia Rosa ML, et al. Relationship between ultra-processed foods and metabolic syndrome in adolescents from a Brazilian Family Doctor Program. Public Health Nutr 2012;15:1-6.

8. Mendonça RD, Pimenta AM, Gea A, et al. Ultraprocessed food consumption and risk of overweight and obesity: the University of Navarra Follow-Up (SUN) cohort study. Am J Clin Nutr 2016;104:1433-40.

9. Rauber F, Campagnolo PD, Hoffman DJ, et al. Consumption of ultraprocessed food products and its effects on children's lipid profiles: a longitudinal study. Nutr Metab Cardiovasc Dis 2015;25:116-22.

10. Flegal KM, Carroll MD, Kit BK, et al. Prevalence of obesity and trends in the distribution of body mass index among us adults, 1999-2010. JAMA 2012;307:491-7.

11. Murray CJ, Atkinson $\mathrm{C}$, Bhalla $\mathrm{K}$, et al. The state of US health, 1990-2010: burden of diseases, injuries, and risk factors. JAMA 2013;310:591-608.

12. Hawkes C. Uneven dietary development: linking the policies and processes of globalization with the nutrition transition, obesity and diet-related chronic diseases. Global Health 2006;2:4.

13. Stuckler D, McKee M, Ebrahim S, et al. Manufacturing epidemics: the role of global producers in increased consumption of unhealthy commodities including processed foods, alcohol, and tobacco. PLoS Med 2012;9:e1001235.

14. Martínez Steele E, Popkin BM, Swinburn B, et al. The share of ultraprocessed foods and the overall nutritional quality of diets in the US: evidence from a nationally representative cross-sectional study. Popul Health Metr 2017;15:6.

15. Johnson CL, Paulose-Ram R, Cl O, et al. National Health and Nutrition Examination Survey: Analytic guidelines, 1999-2010. National Center for Health Statistics 2013.

16. Centers for Disease Control and Prevention (CDC). NC for HS (NCHS. National Health and Nutrition Examination Survey. Measuring Guides for the Dietary Recall Interview. 2015. https://www.cdc.gov/ nchs/nhanes/measuring_guides_dri/measuringguides.htm

17. U.S. Census Bureau, Population Division,Fertility \& Family Statistics Branch. Current Population Survey: definitions and explanations. 2004. http://www.census.gov/population/www/cps/cpsdef.html (accessed 18 Aug 2017).

18. Adams J, White M. Characterisation of UK diets according to degree of food processing and associations with socio-demographics and obesity: cross-sectional analysis of UK National Diet and Nutrition Survey (2008-12). Int J Behav Nutr Phys Act 2015;12:160.
19. Moubarac JC, Batal M, Louzada ML, et al. Consumption of ultra-processed foods predicts diet quality in Canada. Appetite 2017;108:512-20.

20. Cediel G, Reyes M, da Costa Louzada ML, et al. Ultra-processed foods and added sugars in the Chilean diet (2010). Public Health Nutr 2017.9.

21. Marrón-Ponce JA, Sánchez-Pimienta TG, Louzada M, et al. Energy contribution of NOVA food groups and sociodemographic determinants of ultra-processed food consumption in the Mexican population. Public Health Nutr 2018.21.

22. Julia C, Martinez L, Allès B, et al. Contribution of ultra-processed foods in the diet of adults from the French NutriNet-Santé study. Public Health Nutr 2018.21.

23. Wang DD, Leung CW, Li Y, et al. Trends in dietary quality among adults in the United States, 1999 through 2010. JAMA Intern Med 2014;174:1587-95

24. Poti JM, Popkin BM. Trends in energy intake among US children by eating location and food source, 1977-2006. J Am Diet Assoc 2011;111:1156-64.

25. Wang DD, Li Y, Chiuve SE, et al. Improvements In US diet helped reduce disease burden and lower premature Deaths, 1999-2012; Overall Diet Remains Poor. Health Aff 2015;34:1916-22.

26. Reformulation SG. fortification and functionalization: big food corporations' nutritional engineering and marketing strategies. $J$ Peasant Stud 2016;43:17-37.

27. Zagorsky JL, Smith PK. The association between socioeconomic status and adult fast-food consumption in the U.S. Econ Hum Biol 2017;27:12-25.

28. Boyland EJ, Nolan S, Kelly B, et al. Advertising as a cue to consume: a systematic review and meta-analysis of the effects of acute exposure to unhealthy food and nonalcoholic beverage advertising on intake in children and adults. Am J Clin Nutr 2016;103:519-33.

29. Kubik MY, Wall M, Shen L, et al. State but not district nutrition policies are associated with less junk food in vending machines and school stores in US public schools. J Am Diet Assoc 2010;110:1043-8.

30. Ford CN, Slining MM, Popkin BM. Trends in dietary intake among US 2- to 6-year-old children, 1989-2008. J Acad Nutr Diet 2013;113:35-42.

31. Kirkpatrick SI, Dodd KW, Reedy J, et al. Income and race/ethnicity are associated with adherence to food-based dietary guidance among US adults and children. J Acad Nutr Diet 2012;112:624-35.

32. Virudachalam S, Long JA, Harhay MO, et al. Prevalence and patterns of cooking dinner at home in the USA: National Health and Nutrition Examination Survey (NHANES) 2007-2008. Public Health Nutr 2014;17:1022-30.

33. Batis C, Hernandez-Barrera L, Barquera S, et al. Food acculturation drives dietary differences among Mexicans, Mexican Americans, and Non-Hispanic Whites. J Nutr 2011;141:1898-906.

34. Bittman M. Is junk food really cheaper? The New York Times 2011. http://www.nytimes.com/2011/09/25/opinion/sunday/is-junk-foodreally-cheaper.html (accessed 6 Oct 2017).

35. Rao M, Afshin A, Singh G, et al. Do healthier foods and diet patterns cost more than less healthy options? A systematic review and metaanalysis. BMJ Open 2013;3:e004277.

36. Kern DM, Auchincloss AH, Robinson LF, et al. Healthy and unhealthy food prices across neighborhoods and their association with neighborhood socioeconomic status and proportion black/hispanic. J Urban Health 2017;94:494-505.

37. Becker GS. A theory of the allocation of time. Econ $J$ 1965;75:493-517.

38. U.S. Department of Health \& Human Services. Poverty guidelines, research, and measurement. Washington, DC: U.S. Department of Health \& Human Services, 2012. 